\title{
Anti-Aging oder Well-Aging?
}

Anti-Aging ist ein typischer Modebegriff, der schon in sich nicht stimmig ist. Eine Nichtalterung ist bei lebenden Organismen unmöglich. Vielmehr erlauben natürliche Maßnahmen wie gesundheitsförderliche Ernährungsweise, körperliche Aktivität, Stressvermeidung, ausreichender Schlaf aber auch medizinische Therapien wie Hormonersatz ein so genanntes WellAging. Diätetik im humanistischen Sinne bedeutet nichts anderes als gesunde Lebensführung, und eine solche trägt zu einem gesunden Alterungsprozess entscheidend

In Deutschland ist bei. Im Bereich der Mikronährdie Zufuhr von stoffsubstitution muss es auch Mikronährstoffen in Deutschland zu einem nicht optimal Paradigmenwechsel kommen, da die Menschen hierzulande anders essen als sie sich ernähren sollten. Eine Reihe von Untersuchungen belegt, dass in Deutschland die Zufuhr von einigen Mikronährstoffen nur suboptimal ist. Bisher ist es nachweislich nicht gelungen, das Ernährungsverhalten der Bevölkerung in die richtige Bahn zu lenken. Die Zufuhr bestimmter Nahrungsinhaltsstoffe trägt zum Well-Aging bei. Im Gegensatz zu den USA spielt im deutschsprachigen Raum die Nahrungsergänzung oder der Einsatz von ergänzenden bilanzierten Diäten (Food for Special Medical Purpose) eine untergeordnete Rolle. Hier stellt sich die Frage, ob es nicht sinnvoll sein kann, wenn spezifische Mikronährstoffe in besonderen Lebenssituationen ihren Einsatz finden - idealerweise sollte dabei ein therapeutisches Team, dem Arzt, Apotheker und Ernährungsfachkraft angehören, beraten und ggf. überwachen. Dabei geht der Well-Aging-Gedanke aber weit über die Einnahme von Antioxidantien oder anderen Mikronährstoffen hinaus. Ein gesunder Alterungsprozess setzt insbesondere ein gesundes Körpergewicht voraus. Eine Körperfettreduktion bei Übergewicht oder Adipositas scheint die wichtigste Maßnahme für einen gesunden Alterungsprozess darzustellen. Von besonderer Bedeutung ist, dass sowohl ein Untergewicht als auch ein Übergewicht »unge- sund «sind. Gerade Senioren weisen nach den Daten des letzten Mikrozensus des statistischen Bundesamtes aus dem Jahre 1997 mehr als doppelt so häufig einen BMI von weniger als 18,5 auf. Frauen sind doppelt so häufig untergewichtig wie Männer. Sozioökonomische, psychische und physische Faktoren führen im mittleren Erwachsenenalter oftmals zu einer qualitativen Verschlechterung des Ernährungsverhaltens, wobei die quantitative Seite - also die Kalorienzufuhr insgesamt - weniger Veränderung zeigt. Der physiologische Rückgang von aktiver Körpersubstanz, v.a. der Muskulatur, führt im Zusammenhang mit einer positiven Energiebilanz bei weit mehr als der Hälfte der Erwachsenen zu Übergewicht und Adipositas. Es zeigt sich, dass beispielsweise nur 15 Prozent der Adipösen eine normale Lebenserwartung haben. Eine gesund-erhaltende Ernährungsweise bedeutet eine isokalorische Kost, die einen niedrigen glykämischen Index und eine niedrige glykämische Ladung, eine geringe Zufuhr gesättigter Fettsäuren aber ausreichend ungesättigte Fettsäuren - insbesondere Omega-3-Fettsäuren sowie reichlich Nahrungsfasern aufweist. Zu empfehlen sind insbesondere grünblättrige Gemüse und Kaltwasserfisch. Nahrungsergänzungsmittel sollten möglichst sekundäre Pflanzenstoffe einschließen und die Gabe von ergänzenden bilanzierten Diäten sollte unter der Aufsicht von Ernährungsmedizinern und nach Beratung durch qualifizierte Ernährungsfachkräfte (Diätassistenten und Diplom Oecotrophologen) stattfinden.

\section{Jur (bour Gord Gaite}

Sven-David Müller

Hinweis: Die deutsche Schreibweise der beiden parallel vorkommenden englischen Wörter anti-aging und antiageing ist noch nicht abschließend festgelegt. Diese Zeitschrift verwendet bis auf weiteres »Anti-Aging « 